\title{
Digestibilidade Determinada pelo Método Indireto Usando o n-alcano $\mathbf{C}_{35}{ }^{1}$
}

\author{
Dimas Estrásulas de Oliveira², Ênio Rosa Prates ${ }^{3}$, Maria do Carmo R. Peralba ${ }^{4}$
}

\begin{abstract}
RESUMO - Foi feito um trabalho de extração e análise de n-alcanos com o objetivo de investigar o uso do n-alcano $\left(\mathrm{C}_{35}\right)$ presente nas forragens como um indicador interno para determinação de digestibilidade. Foram utilizadas amostras de experimentos de digestibilidade convencional realizados previamente entre os anos de 1978 e 1982. OC $_{35}$ subestimou as digestibilidades da matéria seca e orgânica, apresentando diferença média, em relação ao método in vivo, de $4,68( \pm 0,69)$ e $4,43( \pm 0,85)$ unidades percentuais, respectivamente. O coeficiente de regressão não diferiu da unidade, indicando que os métodos foram equivalentes, havendo, porém, a necessidade de um fator de correção. Os coeficientes de correlação para a matéria seca e orgânica, observados entre os dois métodos, foram 0,87 e 0,85 , respectivamente. $\mathrm{OC}_{35}$ apresentou recuperação fecal média de $91,76 \%( \pm 7,94)$, que contribuiu para o vício da técnica.
\end{abstract}

Palavras-chave: cromatografia gasosa, digestibilidade, gramíneas tropicais, hidrocarbonetos, indicadores

\section{Digestibility Determinate by Indirect Method Using $C_{35}$ n-Alkane}

\begin{abstract}
An experiment was conducted, on extraction and analysis of n-alkanes, to investigate the use of n-alkane $\left(\mathrm{C}_{35}\right)$ present in forages as an internal marker for the digestibility determination. Samples of forages and feces were taken from conventional digestibility trials, which were previous conducted from 1978 to 1982 . The $\mathrm{C}_{35}$ underestimated the dry matter and organic matter digestibilities, presenting an average difference in relation to in vivo of $4.68( \pm 0.69)$ and $4,43( \pm 0.85)$ perceptual units, respectively. The coefficient of regression did not differ from the unit, indicating that both methods were equivalent, having, however, the necessity of a correction factor. The coefficients of correlation observed for the dry and organic matter between the two methods were 0.87 and 0.85 , respectively. The average fecal recovery of $\mathrm{C}_{35}$ was $91.76 \%$ ( \pm 7.94$)$, and this contributed to the bias of the method.
\end{abstract}

Key Words: gas chromatography, digestibility, tropical grasses, hydrocarbons, markers

\section{Introdução}

A cera das plantas possui em sua composição hidrocarbonetos alifáticos saturados (n-alcanos), com comprimentos da cadeia de carbono variando de $\mathrm{C}_{18}$ a $\mathrm{C}_{37}$, havendo predominância dos de cadeia ímpar sobre os pares (CHIBNALL et al., 1934; HAMILTON e POWER, 1969; TULLOCH, 1981; e OLIVEIRA et al., 1997).

ORÓ et al. (1965) relaram a existência de grande similaridade entre o perfil de n-alcanos extraídos das fezes de bovinos e o da forragem consumida (Medicago arabica, L.). Em ambos os materiais, nalcanos de $\mathrm{C}_{18}$ a $\mathrm{C}_{35}$ foram encontrados, predominando $\mathrm{C}_{29}, \mathrm{C}_{31}$ e $\mathrm{C}_{33}$, destes o $\mathrm{C}_{31}$ aparece em maior quantidade.

Trabalhando com ovinos alimentados com uma dieta de azevém (Lolium multiflorum, L. ) e trevo branco (Trifolium repens, L.), MAYES et al. (1984) constataram que os n-alcanos de cadeia longa pode- riam ser úteis como marcadores para estimar a digestibilidade de forragens. Os mesmos autores concluíram, também, que houve progressivo aumento na recuperação fecal, à medida que se elevou o comprimento da cadeia carbonada dos n-alcanos, com recuperação do $C_{35}$ de $97,5 \%$, e sugeriram seu uso como indicador interno de digestibilidade.

DOVE et al. (1990), trabalhando com ovelhas lactantes, estimaram o consumo de forragem (Lolium perenne, L.) por intermédio de estimativas de digestibilidade obtidas pelo método in vitro ou com o n-alcano $\mathrm{C}_{35}$ e concluíram que as estimativas de consumo com base na digestibilidade com o $\mathrm{C}_{35}$ foram mais exatas.

No Rio Grande do Sul e em grande parte do Brasil, onde as criações de bovinos de corte e ovinos desenvolvem praticamente todo o seu ciclo de produção sob condições extensivas de pastejo, há necessidade de se encontrarem técnicas adequadas e confiáveis para a determinação do consumo e da

\footnotetext{
1 Parte da Dissertação de Mestrado do primeiro autor apresentada à UFRGS.

2 Zootecnista, Aluno do Curso de Doutorado em Ciência Animal e Pastagens da ESALQ-USP, Piracicaba, SP. Email: deolivei@carpa.ciagri.usp.br

3 Professor do Curso de Pós-graduação em Zootecnia da UFRGS, Bolsista do CNPq. E.mail: erprates@orion.ufrgs.br

4 Professor Adjunto do Instituto de Química da UFRGS, Bolsista do CNPq. E.mail: mcarmo@if.ufrgs.br
} 
digestibilidade das forragens. Assim, considerando a escassez de informações a respeito de novos indicadores, o objetivo deste trabalho foi avaliar o uso do $\mathrm{n}$-alcano $\mathrm{C}_{35}$ como indicador interno de digestibilidade, em ruminantes.

\section{Material e Métodos}

O experimento foi realizado no Laboratório de Nutrição Animal do Departamento de Zootecnia da Faculdade de Agronomia e no Laboratório de Cromatografia do Instituto de Química da Universidade Federal do Rio Grande do Sul.

Foram utilizadas amostras de fenos e fezes de experimentos dedigestibilidade convencional comovinos, realizados previamente entre os anos de 1978 e 1982, que estavam armazenadas no Laboratório de Nutrição Animal. Os fenos utilizados foram: Milheto I, Milheto II, Setária e Capim-Elefante, cv. Napier, em três alturas de corte: 1,2; 1,5; e 1,8 m (MORATO, 1978).

O método utilizado para a extração de n-alcanos dos fenos e fezes foi o de DILLON e STAKELUM (1990a).

Foi utilizado como indicador interno de digestibilidade on-alcano pentatriacontano $\left(\mathrm{C}_{35} \mathrm{H}_{72}\right)$, visto que, segundo a literatura (MAYES et al., 1984; MAYES et al., 1986a, b; MAYES et al., 1988; DOVE et al., 1989; e DOVE et al., 1990), quanto maior o comprimento da cadeia de carbono, maior é a recuperação fecal, sendo essa característica uma das principais na busca do indicador ideal.

Os procedimentos para determinação do conteúdo de n-alcanos das amostras, as condições cromatográficas usadas, bem como o método usado para identificar individualmente os n-alcanos das amostras de fenos e fezes, são os mesmos detalhados no trabalho de OLIVEIRA et al. (1997).

A digestibilidade da matéria seca foi calculada com base nas concentrações do indicador interno nas fezes e no alimento, segundo PIASENTIER et al. (1989). A digestibilidade da matéria orgânica foi calculada de acordo com SCHNEIDER e FLATT (1975).

Os resultados foram analisados por regressão linear. Para determinar se o coeficiente de regressão (b) era diferente de 1 , utilizou-se o teste t. $\mathrm{O}$ vício associado à técnica foi testado por intermédio do teste de $\mathrm{t}$ para comparações emparelhadas, em que a média das diferenças entre as medições in vivo e as estimadas por indicador representa a estimativa do vício associado à técnica.

Para analisar a precisão do método do indicador, utilizaram-se os critérios de Theil (1961), citado por TORRES e MARTÍNEZ (1986).

\section{Resultados e Discussão}

Os coeficientes de digestibilidade da matéria seca ematéria orgânicaestimados comon-alcano $\mathrm{C}_{35}\left(\mathrm{CDMS}_{35}\right.$ e $\left.\mathrm{CDMO}_{35}\right)$ e in vivo $\left(\mathrm{CDMS}_{\text {vivo }}\right.$ e $\mathrm{CDMO}_{\text {vivo }}$ ), para os vários fenos, são apresentados na Tabela 1. Também são mostrados o vício da técnica em relação aos valores in vivo, o número de observações que geraram a informação, a equação de regressão entre os métodos e os coeficientes de correlação.

O método do n-alcano $\mathrm{C}_{35}$ como indicador subestimou as digestibilidades da matéria seca e orgânica em 4,68 e 4,43 unidades percentuais, respectivamente $(\mathrm{P}<0,01)$, mas os coeficientes de correlação da matéria seca e matéria orgânica obtidos podem ser considerados bons entre as medidas com indicador e in vivo, sendo estes altamente significativos $(\mathrm{P}<0,01)$.

Os interceptos das equações para matéria seca e matéria orgânica diferiram de zero $(\mathrm{P}<0,01$ e $\mathrm{P}<0,05)$, respectivamente, porém os coeficientes de regressão não apresentaram diferenças significativas de 1 , podendo-se interpretar que os métodos são equivalentes, mas havendo necessidade de se aplicar um fator de correção constante. Não se conseguiu utilizar um fator de correção, devido ao moderado ajuste das equações de regressão linear para matéria seca e matéria orgânica $\left(\mathrm{r}^{2}=\right.$ 0,76 e $\left.\mathrm{r}^{2}=0,73\right)$, respectivamente. NARVÁEZ e LASCANO (1989), comparando métodos in vitro de determinação da digestibilidade com espécies forrageiras tropicais, também encontraram resultados semelhantes com relação aos interceptos a e aos coeficientes de regressão b entre os dois métodos, em que um foi considerado padrão. Esses autores sugeriram o uso de fatores de correção, devido ao ótimo ajuste do modelo linear $\left(\mathrm{r}^{2}\right.$ variando de 0,87 a 0,98$)$.

POTT et al. (1978) compararam a digestibilidade in vitro com in vivo, usando 32 plantas forrageiras, e obtiveram coeficientes de correlação de 0,87 e 0,88 para matéria seca e matéria orgânica, sendo esses valores, respectivamente, iguais e maiores que os alcançados neste trabalho (Tabela 1). PIASENTIER et al. (1989) trabalharam com uma gramínea, Dactylis glomerata L., e compararam os valores de digestibilidade da matéria seca in vivo obtidos com ovinos com os resultantes do uso dos n-alcanos $\mathrm{C}_{31}$ e $\mathrm{C}_{33}$ como indicadores internos, e encontraram coeficientes de correlação de 0,80 e 0,84 com errospadrão das estimativas de 2,94 e 2,64 pontos percentuais. 
Tabela 1- Coeficientes de digestibilidade da matéria seca e orgânica estimados com n-alcano $\mathrm{C}_{35}\left(\mathrm{CDMS}_{35}{\text { e } C D M O_{35}}\right)$ e in vivo $\left(\mathrm{CDMS}_{\text {vivo }}\right.$ e $\left.\mathrm{CDMO}_{\text {vivo }}\right)$, vícios das estimativas (D), equações de regressão e coeficientes de correlação $(r)$

Table 1 - The coefficients of digestibility for dry and organic matter, estimated with $C_{35}$ n-alkane $\left(C D M S_{35}\right.$ and $\left.C D M O_{35}\right)$ and in vivo $\left(C D M S_{\text {vivo }}\right.$ and $\left.C D M O_{\text {vivo }}\right)$, bias estimate $(D)$, regression equation and correlation coefficients $(r)$

\begin{tabular}{|c|c|c|c|c|c|c|}
\hline$X$ & $\mathrm{Y}$ & $\hat{\mathrm{D}}$ & OBS & $\begin{array}{c}\text { Regressão } \\
\text { Regression }\end{array}$ & $b \neq 1$ & $\mathrm{r}$ \\
\hline $\begin{array}{c}\mathrm{CDMS}_{35} \\
45,86\end{array}$ & $\begin{array}{c}\mathrm{CDMS}_{\text {vivo }} \\
50,54\end{array}$ & $-4,68 * *$ & 29 & $\hat{Y}=11,88+0,84 X$ & $\mathrm{~ns}$ & $0,87 * *$ \\
\hline $\begin{array}{c}\mathrm{CDMO}_{35} \\
49,29\end{array}$ & $\begin{array}{c}\mathrm{CDMO}_{\text {vivo }} \\
53,72\end{array}$ & $-4,43 * *$ & 29 & $\hat{Y}=12,96+0,82 X$ & $\mathrm{~ns}$ & $0,85^{* *}$ \\
\hline $\begin{array}{ll}* * & (P<0,01 \\
\text { ns } & \text { Não-sig } \\
\star * & (P<.01) . \\
\text { NS } & \text { Not signi }\end{array}$ & ativo. & & & & & \\
\hline
\end{tabular}

Neste trabalho, o coeficiente de correlação obtido entre a digestibilidade in vivo da matéria seca e a estimada com o $\mathrm{C}_{35}$ foi maior que os coeficientes de correlação encontrados pelo autor citado, usando os nalcanos $\mathrm{C}_{31}$ e $\mathrm{C}_{33}$ para a mesma medida, certamente devido ao fato de a recuperação fecal do $C_{35}(91,76 \%)$ ter sido bem superior às do $\mathrm{C}_{31}$ e $_{33}, 79,8$ e 83,4\%, respectivamente, obtidas pelo referido autor.

OHAJURUKA e PALMQUIST (1991), trabalhando com vacas da raça Holandês recebendo duas dietas, uma de feno de alfafa e concentrado e outra de feno de gramínea e concentrado, compararam a digestibilidade da matéria seca in vivo com a estimada, por meio de dois indicadores internos, sendo um nalcano, e verificaram que a digestibilidade da matéria seca foi subestimada $(\mathrm{P}<0,05)$ com o n-alcano $\mathrm{C}_{31} \mathrm{H}_{64}$. No mesmo trabalho, a recuperação fecal média do $\mathrm{C}_{31}$, em ambas as dietas, foi menor que $100 \%$, contribuindo para a subestimativa da digestibilidade da matéria seca.

$\mathrm{Na}$ Tabela 2, são apresentadas as proporções do erro nas digestibilidades da matéria seca e orgânica estimadas com o indicador, devido ao vício associado à técnica, à regressão e a causas aleatórias. Ficaram evidentes altos percentuais de erro devido ao vício e a causas aleatórias. $\mathrm{O}$ alto percentual de erro devido ao vício da técnica indica, de forma consistente, a subestimativa da digestibilidade tanto da matéria seca como da matéria orgânica, com o uso do indicador em questão. $\mathrm{O}$ alto percentual de erro devido a causas aleatórias pode ser atribuído à variabilidade inerente do material experimental e, talvez, pudesse ser reduzido aumentando-se o tamanho da amostra. TORRES e MARTÍNEZ (1986) comentaram que boa predição deve apresentar baixo percentual de erro devido ao vício e à regressão, com maior percentual devido a causas aleatórias.
A exatidão da técnica de relação, segundo LE DU e PENNING (1982), é influenciada pelo vício, porque se assume que a recuperação fecal do indicador é $100 \%$, e também, pelo erro aleatório associado com as concentrações do indicador no alimento e fezes, além da possibilidade de haver, também, erro devido à análise química do indicador.

A determinação da digestibilidade usando um indicador por intermédio da técnica de relação considera alguns pressupostos que, quando não acontecem na íntegra, viciam a técnica e colocam dúvida sobre o indicador em questão.

Conforme visto anteriormente, as digestibilidades da matéria seca e matéria orgânica estimadas com o n-alcano $\mathrm{C}_{35}$, como indicador, foram subestimadas de forma significativa, mostrando que o mesmo não apresentou comportamento totalmente aceitável. Provavelmente houve perda variável do n-alcano $\mathrm{C}_{35}$ no

Tabela 2 - Fontes de variação e proporções de erro, devido ao vício, à regressão e a aleatoriedade na digestibilidade da matéria seca (MS) e matéria orgânica (MO) estimada com n-alcano $\mathrm{C}_{35}$ $\left(\mathrm{CDMS}_{35}\right.$ e $\left.\mathrm{CDMO}_{35}\right)$

Table 2 - Sources of variations and error proportions by bias, regression and aleatoriety in the dry matter (DM) and organic matter (OM) digestibilities estimated with $C_{35}$ $n$-alkane $\left(\mathrm{CDMS}_{35}\right.$ and $\left.\mathrm{CDMO}_{35}\right)$

\begin{tabular}{ccc}
\hline $\begin{array}{c}\text { Fonte de variação } \\
\text { Source of variation }\end{array}$ & $\begin{array}{c}\text { MS }(\%) \\
D M\end{array}$ & $\begin{array}{c}\text { MO }(\%) \\
O M\end{array}$ \\
\hline $\begin{array}{c}\text { Vício } \\
\text { Bias } \\
\text { Regressão } \\
\begin{array}{l}\text { Regression } \\
\text { Aleatoriedade } \\
\text { Aleatoriety }\end{array}\end{array}$ & 52,11 & 48,19 \\
\hline
\end{tabular}


trato digestivo, o qual apresentou recuperação média de $91,76 \%( \pm 7,94)$, para os vários fenos em que se estimaram as digestibilidades da matéria seca e matéria orgânica. Esta falta de recuperação do $\mathrm{C}_{35}$ (8,24 unidades percentuais), consideravelmente alta, certamente contribuiu para as subestimativas das digestibilidades da matéria seca e matéria orgânica (Figura 1).

A recuperação média obtida do $\mathrm{C}_{35}$ foi menor que as obtidas por MAYES et al. (1984), de 97,5\%; MAYES et al. (1986a), de 93,1\%; MAYES et al. (1986b), de 93,4\%; MAYES et al. (1988), de 95,3\%; e STAKELUM e DILLON (1990), de 94\%.

Recuperações fecais do $\mathrm{C}_{35}$ inferiores às obtidas neste trabalho foram apresentadas por KAFILZADEH e PARKER (1990), de 85\%; DILLON e STAKELUM (1990b), de 77,8\%; e VULICH et al. (1991), de $91 \%$.

A literatura cita que há aumento na recuperação fecal dos n-alcanos, à medida que se eleva o comprimento da cadeia carbonada, sendo que, neste trabalho, também se verificou esse comportamento, obtendo-se coeficiente de correlação $(r=0,78)$ entre as duas variáveis, o qual não foi maior em razão de a recuperação fecal do $\mathrm{C}_{31}(91,51 \%)$ ter sido maior que a do $C_{33}(89,79 \%)$. Maior grau de associação entre essas duas variáveis foram obtidos por MAYES et al. (1984) $r=0,96$; MAYES et al. (1986a, b) $r=0,96$ e PIASENTIER et al. (1989) $r=0,99$. Cabe ressaltar, ainda, que as recuperações fecais do $\mathrm{C}_{29}(74,22 \%)$ e $\mathrm{C}_{33}(89,79 \%)$ registradas nesta pesquisa são bem próximas às obtidas por MAYES et al. (1984) $\mathrm{C}_{29}=72,2 \%$ e $\mathrm{C}_{33}=90,9 \%$; MAYES et al. (1986) $\mathrm{C}_{29}=74,5 \%$ e $\mathrm{C}_{33}=89,1 \%$ e maiores que as encontradas por PIASENTIER et al. (1989), 72,1 e 83,4\%, respectivamente, para os mesmos referidos n-alcanos.

Um fator que pode contribuir nos erros, quando se calcula a recuperação fecal, é a amostragem da forragem consumida e das fezes produzidas. Sabe-se que a seleção do alimento feita pelos animais é maior sob condições de pastejo, mas não se pode descartar que isso não ocorra com animais mantidos em gaiolas metabólicas. Este fato foi comprovado por KENNEY e BLACK (1984), em um estudo sobre fatores que influem na seleção da dieta por ovinos mantidos em gaiolas metabólicas.

Mesmo com uma dieta monoespecífica, quando os animais selecionam partes da planta com concentrações diversas de n-alcanos (HERBIN e ROBINS, 1969; TULLOCH, 1973; LAREDO et al. 1991) e não há sobras do alimento para análise e possível corre-

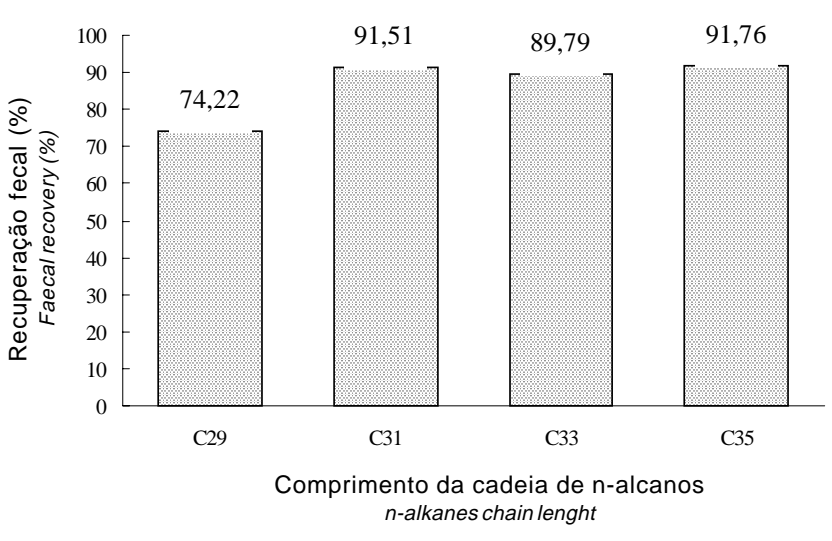

Figura 1 - Recuperações fecais dos n-alcanos $\mathrm{C}_{29}$ ao $\mathrm{C}_{35}$ dos diversos fenos estudados.

Figure 1 - Fecal recoveries of $C_{29}$ to $C_{35}$ n-alkanes of various studied hays.

ção, certamente haverá fezes em que as concentrações de alcanos não irão corresponder àquelas da amostra do alimento.

As amostras disponíveis para este estudo foram de alimentos e fezes, de modo que não foi possível corrigir os valores das digestibilidades estimadas pela relação.

Diante do exposto, seria recomendável que fossem feitos outros experimentos envolvendo o uso de nalcanos como indicadores de digestibilidade, haja vista que o uso destes é recente e, até a presente data, não há trabalhos publicados nessa área, no Brasil, havendo, portanto, escassez de dados sobre o assunto.

\section{Conclusões}

$\mathrm{O}$ n-alcano $\mathrm{C}_{35}$ como indicador subestimou as digestibilidades da matéria seca e matéria orgânica, devido ao vício da técnica causado pela recuperação fecal média inferior a $100 \%$.

Há progressivo aumento na recuperação fecal dos n-alcanos, à medida que se eleva o comprimento da cadeia de carbono.

\section{Referências Bibliográficas}

CHIBNALl, A.C., PIPER, S.H., POLLARD, A. et al. 1934. The constitution of the primary alcohols, fatty acids and paraffins present in plant and insect waxes. Biochemistry J., 28:2189-2208.

DILLON, P., STAKELUM, G. The analysis of n-alkanes in faeces and herbage. In: EUR. GRAZING WORKSHOP, 7, 1990, Wageningen. Proceedings... Wageningen, 1990a. 
852 Rev. bras. zootec.

DILLON, P., STAKELUM, G. Dosed and herbage alkanes for predicting silage intake with dairy cows: The effect of concentrate type and level of feeding. In: EUR. GRAZING WORKSHOP, 7, 1990, Wageningen. Proceedings... Wageningen, 1990b.

DOVE, H., MAYES, R.W., FREER, M., et al. Faecal recoveries of the alkanes of plant cuticular waxes in penned and grazing sheep. In: INTERNATIONAL GRASSLAND CONGRESS, 16, 1989, Nice. Proceedings... Nice, p.1093-1094, 1989.

DOVE, H., MILNE, J.A., MAYES, R.W. 1990. Comparison of herbage intakes estimated from "in vitro" or alkane-based digestibilities. Proceedings New Zealand Soc. Anim. Prod., 50:457-459.

HAMILTON, R.J., POWER, D.M. 1969. The chemical composition of the surface wax of Lolium perenne. Phytochemistry, 8(9):1771-1775.

HERBIN, G.A., ROBINS, P.A. 1969. Patterns of variation and development in leaf wax alkanes. Phytochemistry, 8(10):1985-1998.

KAFILZADEH, F., PARKER, D.S. The use of n-alkanes as indigestible markers in studies on intestinal digestion in sheep. In: MEETING BRITISH SOCIETY ANIMAL PRODUCTION, 100, London, 1990. Proceedings... London, v.50, p.578-579.

KENNEY, P.A., BLACK, J.L. 1984. Factors affecting diet selection by sheep. I Potential intake and acceptability of feed. Aust. J. Agric. Res., 35(4):551-563.

LAREDO, M.A., SIMPSON, G.D., MINSON, D.J. et al. 1991. The potential for using n-alkanes in tropical forages as a marker for determination of dry matter by grazing ruminants. J. Agric. Sci., 117(3):355-361.

LE DU, Y.L.P., PENNING, F.D. 1982. Animal based techniques for estimating herbage intake. In: LEAVER, J.D. (Ed.). Herbage intake handbook. Hurley: Brit. Grassl. Soc. p.37-75.

MAYES, R.W., LAMB, C.S., COLGROVE, P.M. The possible use of n-alkanes in herbage as indigestible faecal markers. In: GENERAL MEETING EUROPEAN GRASSLAND FEDERATION, 1984. Proceedings... Nutr. Soc., v.43, n.13, p.39, 1984. (Abstract).

MAYES, R.W., LAMB, C.S., COLGROVE, P.M. 1986a. The use of dosed and herbage n-alkanes as markers for the determination of herbage intake. J. Agric. Sci., 107:161-170.

MAYES, R.W., LAMB, C.S., COLGROVE, P.M. 1986b. Determination of herbage intake of suckling lambs using longchain n-alkanes as markers. Br. Soc. Anim. Prod., 42:457 (Abstract).

MAYES, R.W., LAMB, C.S., COLGROVE, P.M. Digestion and metabolism of dosed even-chain and odd-chain n-alkanes in sheep. In: GENERAL MEETING EUROPEAN GRASSLAND FEDERATION, 12, 1988, Dublin. Proceedings... Dublin, p.159-163, 1988.

MORATO, H.E. Determinação do valor nutritivo do capim elefante (Pennisetum purpureum, Schum.) em três estádios de maturidade através de ensaio de digestibilidade, consumo voluntário e balanço nitrogenado com ovinos. Porto Alegre, RS, UFRGS, 1978. 72p. Dissertação (Mestrado em Zootecnia) - Faculdade de Agronomia/Universidade Federal do Rio Grande do Sul, 1978.

NARVÁEZ, V.N., LASCANO,C. 1989. Digestibilidad in vitro de especies forrajeras tropicales. 1. Comparación de métodos de determinación. Pasturas Tropicales, 11(1):13-17.

OHAJURUKA, O.A., PALMQUIST, D.L. 1991. Evaluation of n-alkanes as digesta markers in dairy cows. J. Anim. Sci., 69(4):1726-1732.

OLIVEIRA, D.E., PRATES, E.R., PERALBA, M.C.R. 1997. Identificação e quantificação de n-alcanos presentes nas ceras de plantas forrageiras. R. Bras. Zootec., 26(5):881-886.

ORÓ, J., NOONER, D.W., WIKSTRÖN, S.A. 1965. Paraffinic hydrocarbons in pasture plants. Science, 147(3659):870873.

PIASENTIER, E., PISON, S., BOVOLENTA, S. 1989. Impiego degli n-alcani megli studi sulla digeribilitá in vivo dei foraggi. Zoot. Nutri. Anim., 15:691-696.

POTT, E.B., PRATES, E.R., LEBOUTE, E.M. 1978. Correlações entre os coeficientes de digestibilidade da matéria seca e entre os da matéria orgânica determinados com animais e por técnica "in vitro". R. Soc. Bras. Zootec., 7(1):26-42.

SCHNEIDER, B.H., FLATT, W.P. 1975. The evaluation of feeds through digestibility experiments. Athens, Univ. Georgia Press. 423p.

STAKELUM, G., DILLON, P. Dosed and herbage alkanes as feed intake predictors with dairy cows: The effect of feeding level and frequency of perennial ryegrass. In: EUROPEAN GRAZING WORSHOP, 7, 1990, Wageningen. Proceedings... Wageningen, 1990.

TORRES, V., MARTÍNEZ, J. 1986. Método visual para estimar la disponibilidad del pasto. I. Estudios de precisión. Rev. Cubana Ciencia Agric., 20(1):1-7.

TULLOCH, A.P. 1973. Composition of leaf surface waxes of triticum species: Variation with age and tissue. Phytochemistry, 12(9):2217-2223.

TULLOCH, A.P. 1981. Composition of epicuticular waxes from 28 genera of Graminae: Differences between subfamilies. Can. J. Bottany, 59(7):1213-1221.

VULICH, S.A., O'RIORDAN, E.G., HANRAHAN, J.P. 1991. Use of n-alkanes for estimation of herbage intake in sheep: accuracy and precision of the estimates. J. Agric. Sci., 116(2):319-323.
Recebido em: 09/06/98 Aceito em: 18/10/99 